\title{
Application of Environmental Scanning Electron Microscopy to the Study of Food Systems
}

\author{
B.L. Thiel, D.J. Stokes and A.M. Donald \\ Polymers and Colloids Group, Cavendish Laboratory, University of Cambridge, Cambridge CB3 \\ OHE, UK
}

The many advantages of Environmental Scanning Electron Microscopy (ESEM) over conventional high-vacuum SEM allow an entirely new role for electron microscopy in the study of food systems. Not only can the environment around the specimen be adjusted to accommodate a far more diverse range of specimens, but the very nature of the investigations possible is greatly expanded as well. It is now possible to study dynamic processes rather than simply imaging static microstructures. There are many parallels between food science and materials science, in that both fields attempt to manipulate microstructures in order to achieve desirable physical properties. Accordingly, in this paper we review a number of our ESEM studies of food systems as seen through the eyes of a materials scientist. The ESEM techniques and methodologies appropriate for the investigation of food systems will be discussed elsewhere. [1]

The most fundamental advantage ESEM offers for the study of foods is the ability to control the hydration state of the specimen. This capability allows the user to prevent dehydration of wet or water-containing samples. This enables the study of cellular materials such as plant parenchymal tissue. For example, we studied the real-time microstructural response of carrot tissue to in situ slicing for samples boiled for different times.[2] These studies allowed us to identify changes in stress transmission and the failure mechanisms of the tissue that take place at a critical amount of cell wall degradation. Figure 1 depicts a scalpel blade cutting through cooked carrot tissue. Still images or even live video can be recorded during mechanical testing, allowing specific events in the load-extension curves to be correlated with structural changes.

In other systems, such as starch-based foods, it is more desirable to control the relative humidity, rather than keeping the material moist. In another set of studies, we were able to examine the effects of relative humidity on the mechanical properties of breadcrumbs. The glass transition of starch shifts over a large range, depending on moisture content, and is part of the physical phenomenon underlying the perceptions of texture and 'staleness'. ESEM investigations clearly illustrated the ductile-brittle transition behaviour for different bread preparations as function of relative humidity. [3] Figure 2 shows the step-wise propagation of a crack at the point of failure of a breadcrumb under tensile loading.

Far more impressive is the ability to stabilise and image entirely liquid specimens, such as colloids, emulsions and other complex fluids that are very prominent in the prepared foods industry.[4] In these cases, secondary electron emission processes only accessible in the ESEM give rise to molecular chemistry based contrast mechanisms. Because such substances are in the liquid state, the high mobility of any created or injected charge carriers prevents uncontrolled charging during the imaging of intrinsically dielectric liquids.[5] Figure 3 shows the emulsion microstructure of mayonnaise. (We note that although uncoated materials can be examined with a field emission high vacuum instrument, wet specimens cannot be examined without cryogenic preparation.) 
Finally, water may be present in the form of ice, such as in the multi-phasic composite microstructure of ice cream. Although it is possible to image ice cream using cryo-SEM, it is often desirable to examine the structure at 'storage conditions', a few degrees below zero. This presents some technical challenges, as a small partial pressure of water vapour is still needed to prevent sublimation of the ice, but there must also be sufficient gas to image the specimen. These obstacles were overcome by mixing a small amount of water vapour with nitrous oxide, allowing high quality images to be recorded, as shown in figure 4 [6].

1) D.J. Stokes, B.L. Thiel and A.M. Donald, elsewhere in this volume.

2) B.L. Thiel and A.M. Donald, Journal of Texture Studies, Vol. 31, 437-455 (2000).

3) D.J. Stokes and A.M. Donald, Journal of Materials Science, Vol.35, 599-607 (2000).

4) B.L. Thiel and A.M. Donald, Journal of Molecular Liquids, Vol. 80, 208-230 (1999).

5) D.J. Stokes, B.L. Thiel, and A.M. Donald, Scanning, Vol. 22, 357-365 (2000).

6) A.L. Fletcher, Ph.D. thesis, University of Cambridge (1997).

7) The funding and support of the BBSRC, the Royal Society, Unilever, FEI, and the Sir Isaac Newton Trust a re gratefully acknowledged.
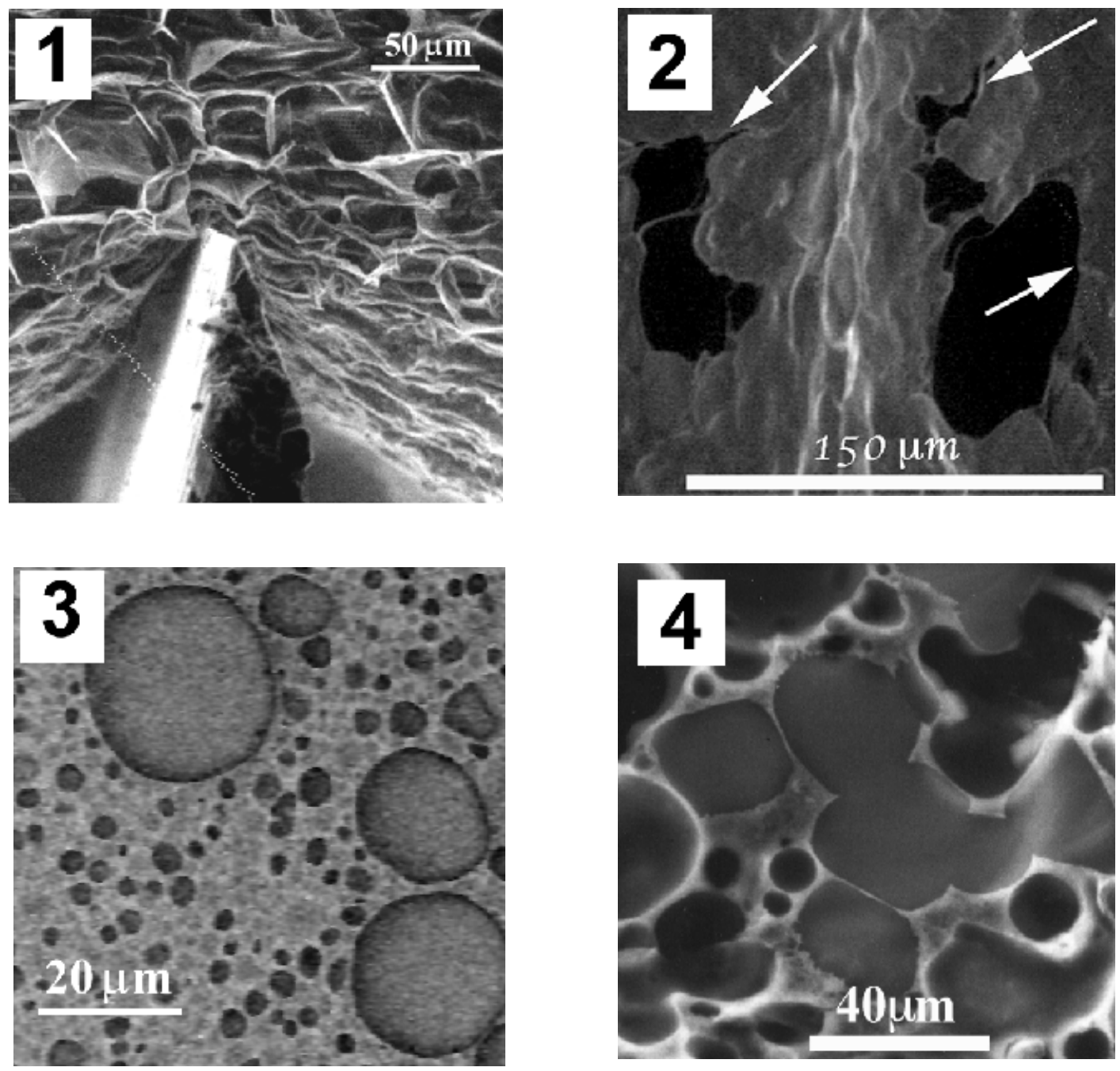